Casos Clínicos

\title{
Hiperplasia pseudoangiomatosa nodular gigante de la mama
}

\author{
Patricia Rubio C. ${ }_{1}$, Fernando Colmenarejo G. ${ }^{1}$, Sofía Hakim A. ${ }^{2}$, Lorena Franco J. ${ }^{1}$, \\ Isabel Vicente $G$. 1 \\ 1 Servicio de Ginecología y Obstetricia, ${ }^{2}$ Servicio de Anatomía Patológica. Hospital Universitario Miguel Servet. \\ Zaragoza, España.
}

\section{RESUMEN}

La hiperplasia pseudoangiomatosa (PASH) es una lesión proliferativa benigna de la mama, poco frecuente, caracterizada por la existencia de lagos pseudovasculares embebidos en una gran proliferación del estroma mamario. Probablemente, el desarrollo de la PASH tenga una influencia hormonal, por lo que típicamente se diagnostica en mujeres en edad fértil. La PASH es un hallazgo histopatológico casual en las piezas quirúrgicas y biopsias mamarias realizadas por otra patología. La presentación clínica en forma de masa palpable es poco frecuente. El principal diagnóstico diferencial debe realizarse con el angiosarcoma de bajo grado. El tratamiento de la PASH nodular es una correcta exéresis quirúrgica asegurando borde sano amplio. El pronóstico es excelente, con un mínimo riesgo de recidiva si se realiza una adecuada cirugía. Se presenta el caso de una mujer de 37 años que acude a consulta por un nódulo mamario de crecimiento rápido.

\section{KEY WORDS: Hiperplasia pseudoangiomatosa, tumor mamario}

\section{SUMMARY}

Pseudoangiomatous stromal hyperplasia (PASH) of the breast is a rare benign proliferative mesenchymal lesion characterized by the presence of open slit like spaces embedded in a hyalinized fibrous stroma. The development of PASH is probably subject to hormonal influence so it typically affects women in the reproductive age group. Pseudoangiomatous stromal hyperplasia is frequently an incidental histologic finding in breast surgeries or biopsies performed for other injuries. In rare cases, it presents as a localized breast mass. The most important differential diagnosis is low-grade angiosarcoma. Tumorous PASH is treated by local surgical excision with clear margins. The prognosis is excellent, with minimal risk of recurrence after adequate surgery. The presented case was a 37 -years-old woman who was admitted with a rapidly growing breast tumor.

KEY WORDS: Pseudoangiomatous stromal hyperplasia, breast tumor 


\section{INTRODUCCIÓN}

La hiperplasia pseudoangiomatosa de la mama (PASH) es una entidad benigna caracterizada por la proliferación del tejido conjuntivo del estroma mamario que se distribuye formando pseudocavidades tapizadas por células fusiformes que sugieren una falso crecimiento vascular (1).

Se trata de una patología poco frecuente, de etiología desconocida, que afecta a mujeres premenopáusicas o menopáusicas en tratamiento con terapia hormonal, por lo que se postula su relación con las hormonas sexuales, especialmente con la progesterona $(2,3)$.

La forma de presentación puntual es la más frecuente, apareciendo como hallazgo casual en el $23 \%$ de las piezas de mastectomía y biopsias de mama realizadas por otros motivos. Por ello, se documenta la asociación de la PASH con multitud de procesos, incluidos neoplasias $(1,4)$.

Se expone el caso de una hiperplasia pseudoangiomatosa de la mama de tipo nodular gigante de aparición durante el segundo trimestre de embarazo.

\section{Caso clínico}

Paciente de 37 años, de raza negra, sin antecedentes de interés que consulta por aparición repentina de un nódulo en mama derecha (MD) de crecimiento muy rápido durante el último mes.

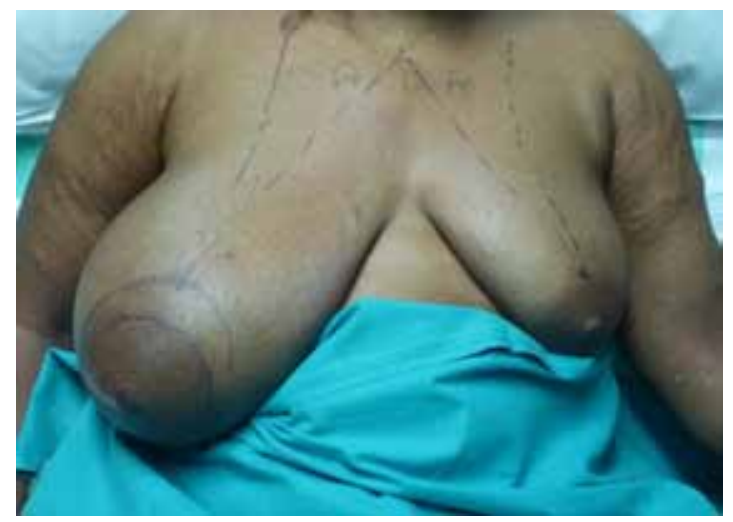

Figura 1. Asimetría mamaria a expensas de voluminosa tumoración de crecimiento muy rápido que ocupa toda la mama derecha.
A la exploración se objetiva una marcada asimetría mamaria a expensas de una tumoración de $20 \mathrm{~cm}$, bien definida, de consistencia firme y bordes lisos que ocupa toda la MD. No hay afectación cutánea ni del complejo areola-pezón. No se palpan adenopatías sospechosas (Figura1).

En el estudio mamario se informa de una voluminosa masa isodensa y homogénea, pseudoencapsulada de 220 x $180 \mathrm{~mm}$ con aparentes cavidades vasculares que no captan Doppler (Figura 2 y 3). Se toma una biopsia con aguja gruesa $14 \mathrm{G}$ que resulta un tumor bifásico fibroepitelial tipo fibroadenoma vs phyllodes.

Se practica una tumorectomía mediante incisión circular concéntrica periareolar tipo Round Block o Doughnut Mastopexy con el fin de mantener la estética y simetría mamaria, tras la exéresis de una lesión de gran tamaño (Figura 4 y 5). En el lecho quirúrgico queda una amplia cavidad sangrante que exige cuidadosa hemostasia asociando un producto sellante para evitar una cauterización excesiva del fondo y bordes de la herida, apostando por una buena cicatrización y reducción del riesgo de infección sobre grandes escaras de tejido necrótico.

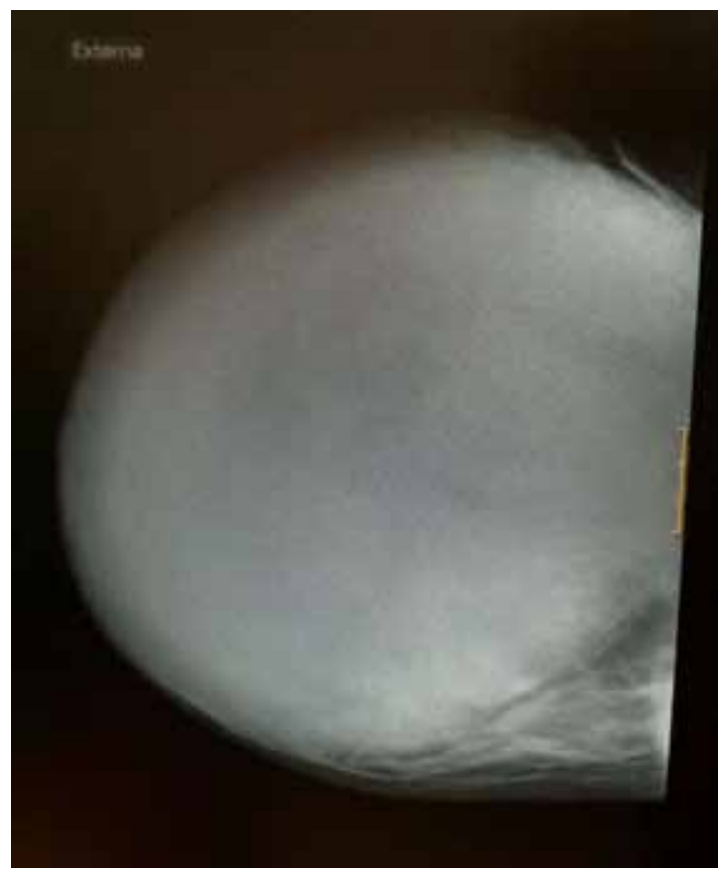

Figura 2. Imagen isodensa y homogénea aparentemente encapsulada. 


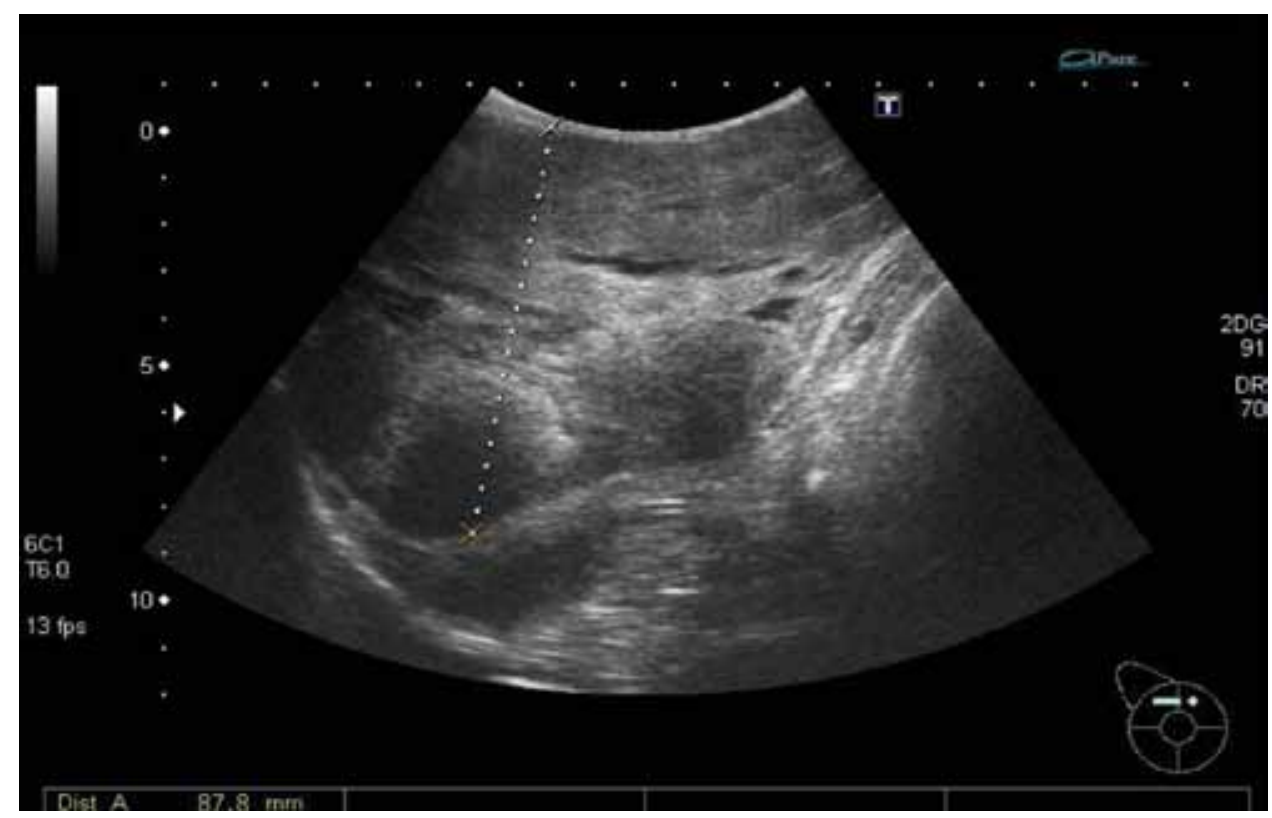

Figura 3. Aparentes cavidades vasculares que no captan Doppler.

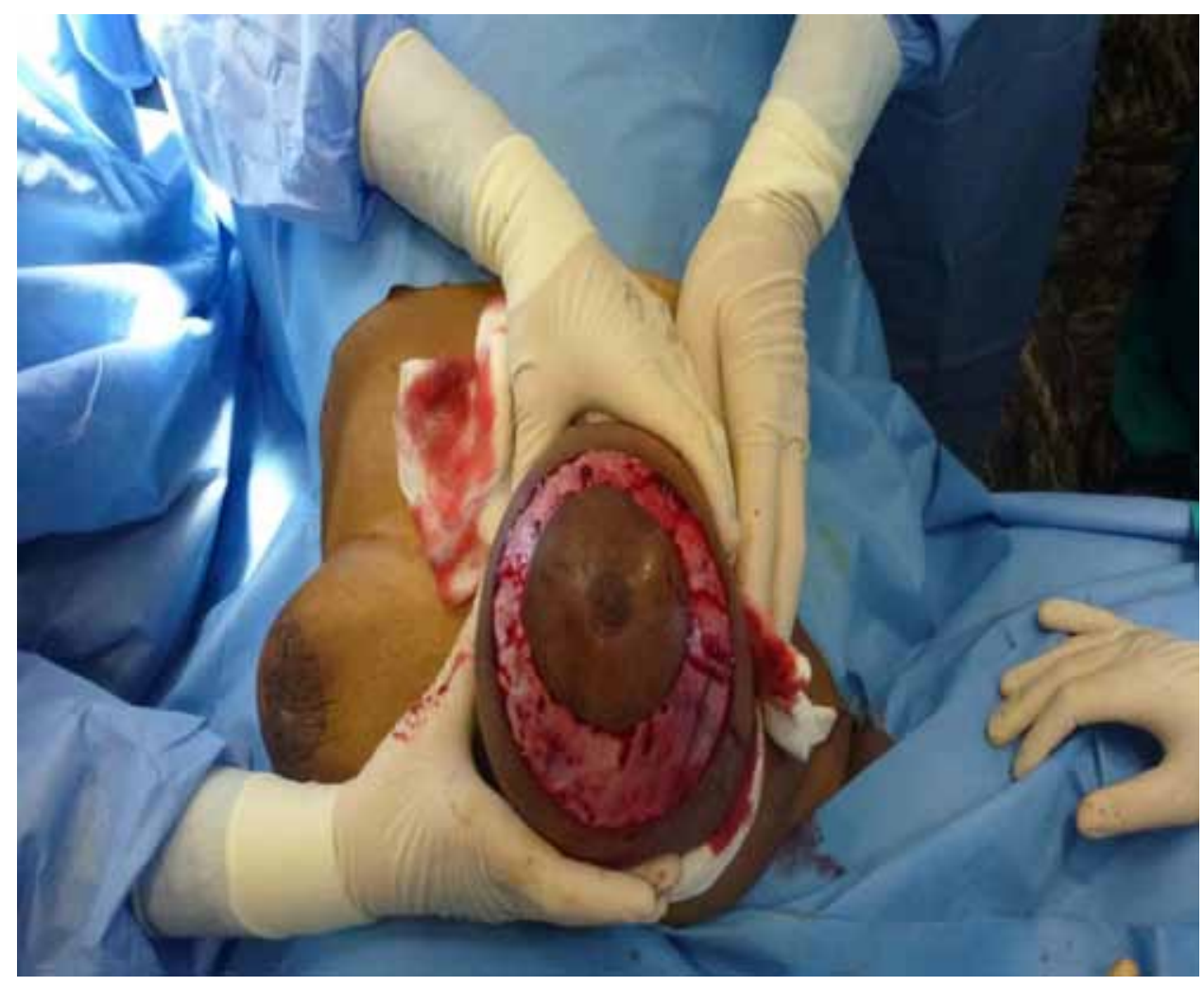

Figura 4. Round Block Technique o Doughnut Mastopexy. 


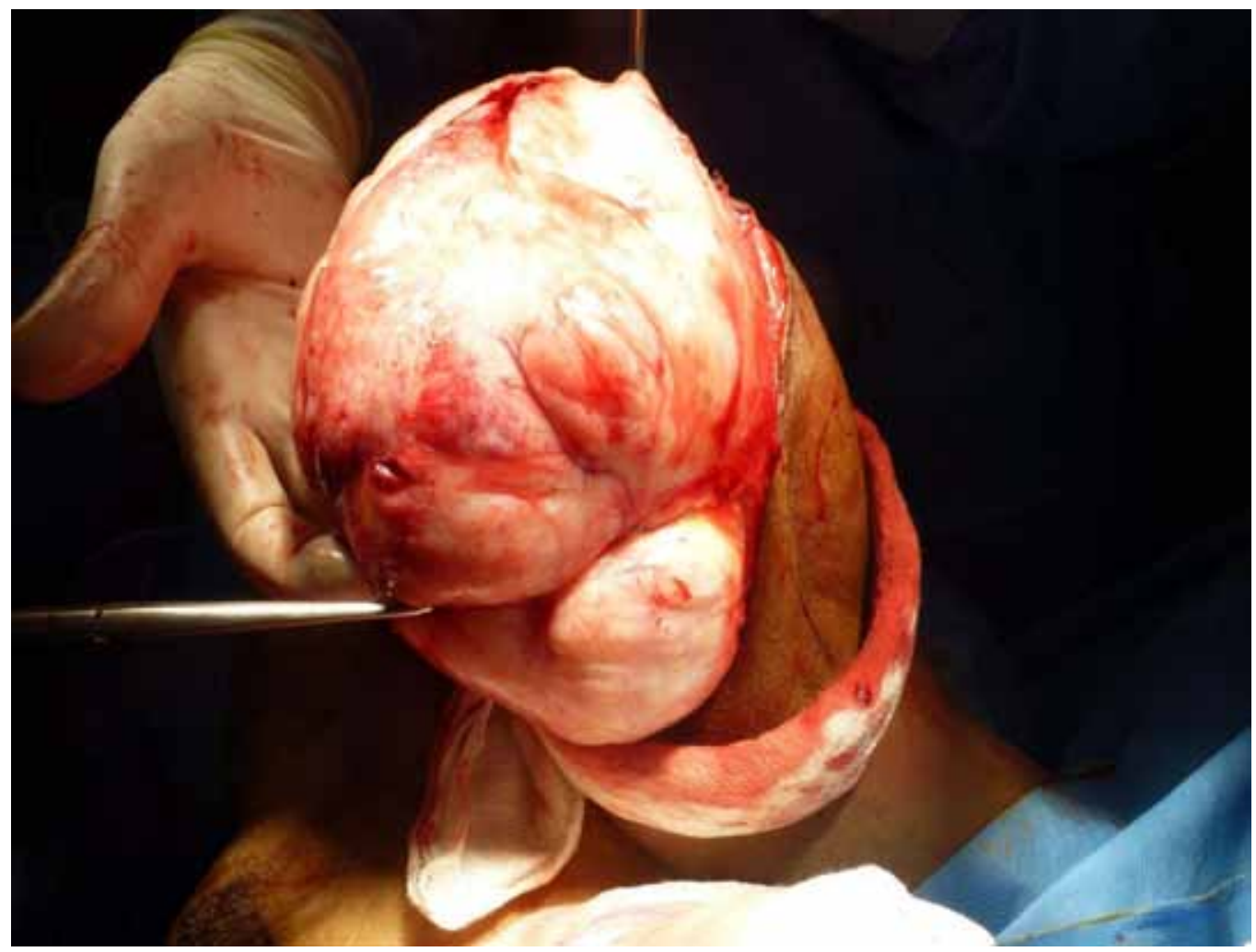

Figura 5. Tumorectomía gigante.

En la descripción macroscópica de la pieza se describe un nódulo regular de $20 \mathrm{~cm}$ de eje mayor, de consistencia elástica, no encapsulado y con abundante vacularización (Figura 6).

El informe anátomo-patológico final diagnostica una hiperplasia pseudoangiomatoide del estroma mamario (Figura 7 y Figura 8).

La recuperación postoperatoria fue rápida y sin incidencias con muy buen resultado estético a las tres semanas de la cirugía (Figura 9).

\section{DISCUSIÓN}

La PASH es una patología de baja prevalencia y etiología sin definir, propia de mujeres jóvenes entre los 30 y 40 años, registrándose algún caso en mujeres menopáusicas bajo terapia hormonal sustitutiva y varones previamente afectos de ginecomastia (1-3).

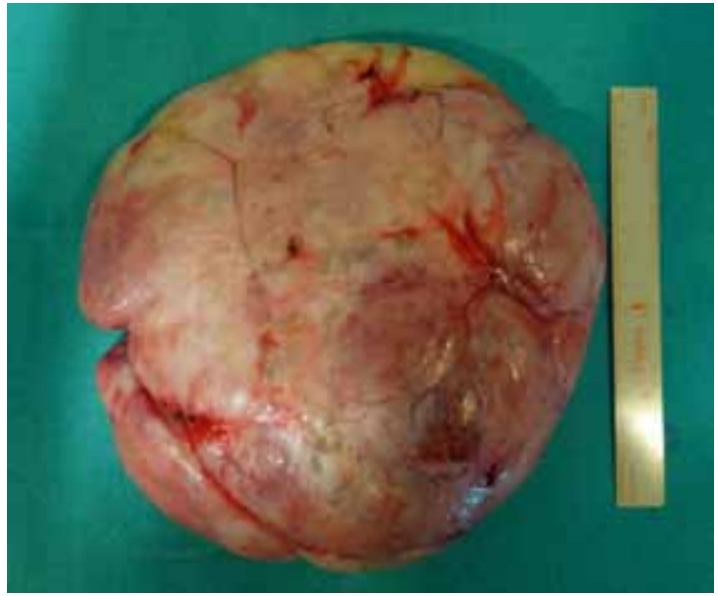

Figura 6. Nódulo regular, de consistencia elástica, no encapsulado con abundante vacularización. 


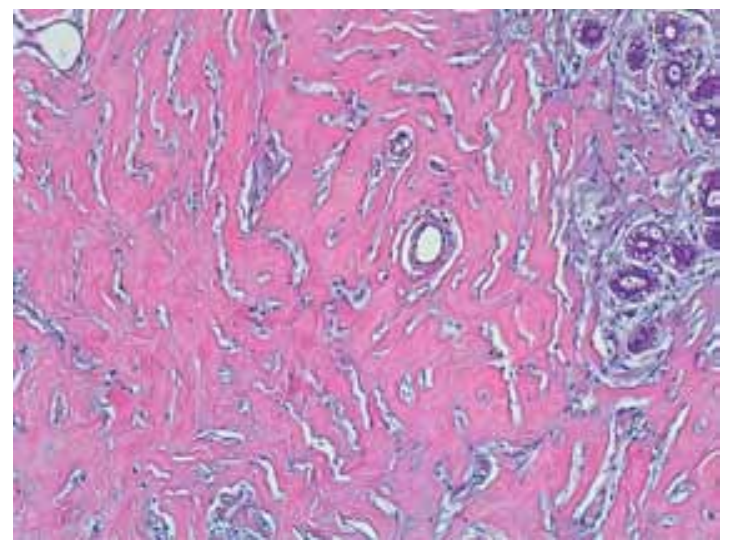

Figura 7. Hematoxilina-Eosina (100X). Estroma fibroso vascularizado denso con presencia de amplias hendiduras anastomosadas simulando espacios vasculares que crecen rodeando estructuras ductales y lobulillares sin alteraciones histopatológicas.

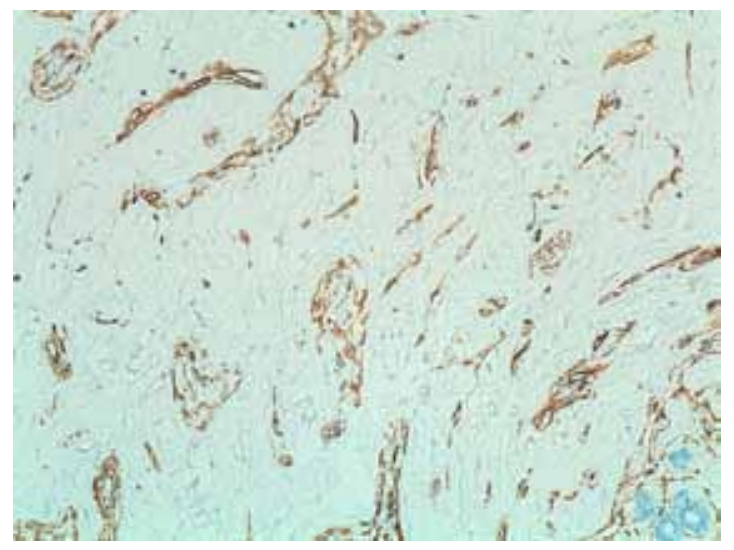

Figura 8. CD34 (100x). Las células del estroma mamario muestran inmunoreactividad para CD34.

El análisis microscópico revela una gran proliferación del tejido conectivo fibroso del estroma mamario que, en su progresión, va formando pseudocavidades confluyentes entre sí, tapizadas por células fusiformes. En los estudios de imagen, esta morfología sugiere falsas dilataciones vasculares que orientan la sospecha diagnóstica hacia tumoraciones de origen vascular como el angioma o angiosarcoma (5). La inmunohistoquímia confirma el origen mesenquimal de estas células, positivas para marcadores estromales como vimentina, actina y CD34, y negativas para CD 31 y Factor VIII propios de los vasos sanguíneos (4).

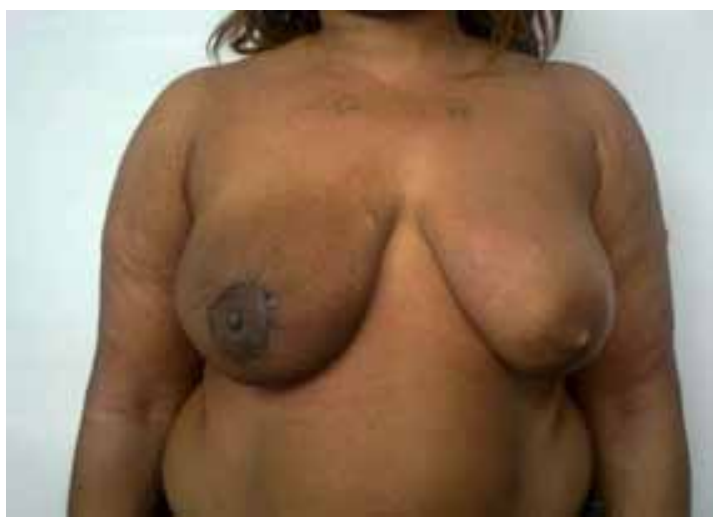

Figura 9. Buen resultado estético a las tres semanas de la cirugía.

La PASH fue descrita por Vuitch y cols en 1986 (6). Se desconoce su etiología exacta, si bien, se postula que pudiera tratarse de una respuesta exagerada y aberrante de los miofibroblastos mamarios a la acción de determinadas hormonas, endógenas o exógenas. La intensa positividad para receptores de progesterona hace pensar que pudiera tratarse del principal agente causal $(1,4)$.

La mayoría de veces la PASH es asintomática y tan siquiera se manifiesta como imagen reseñable en los estudios radiológicos. Habitualmente se trata de un hallazgo casual, en el contexto de una pieza de mastectomía o biopsias de mama realizadas por otra razón, documentándose focos puntuales o dispersos de PASH hasta en un $23 \%$ de los especímenes de mama $(1,4)$.

La presentación nodular es la menos frecuente aunque la más llamativa. Clínicamente aparece como una tumoración única, bien delimitada, de consistencia firme y habitualmente de gran tamaño secundario al rápido crecimiento.

El estudio por imagen revela una masa homogénea, pseudoencapsulada con multitud de cavidades de apariencia vascular que desplaza y comprime el resto de tejido mamario sin infiltrarlo (5). El diagnóstico definitivo debe ser histológico, mediante una biopsia con abundante material o sobre la propia tumorectomía, ya que la escasa incidencia del cuadro puede dejar la PASH fuera del diagnóstico diferencial (1). La hiperplasia pseudoangiomatosa suele ser unilateral y maligniza en muy raras ocasiones.

El tratamiento es quirúrgico mediante la exéresis completa de la tumoración. El pronóstico es excelente con una tasa de recidiva del 15 al $22 \%$ 
asociada a lesiones multicéntricas no resecadas, extirpación incompleta o crecimiento de novo. Por esto, se recomienda el seguimiento de las pacientes a largo plazo. El diagnóstico diferencial debe realizarse con otros procesos proliferativos del estroma como el fibroadenoma y el tumor phyllodes $(1,2,4)$.

\section{CONCLUSIÓN}

La PASH es una entidad anátomo-patológica identificada por casualidad en el $23 \%$ de los especímenes mamarios. La presentación nodular es poco frecuente y llamativa, ya que evoluciona en pocas semanas hasta alcanzar masas de grandes tamaños. Es una patología benigna que afecta a mujeres en edad fértil, como una tumoración mamaria única, lisa, pseudoencapsulada y muy vascularizada, de crecimiento rápido probablemente influido por la progesterona. En el diagnóstico diferencial debe incluirse el tumor phyllodes y el fibroadenoma. El tratamiento es quirúrgico con poca tendencia a la recidiva.

\section{REFERENCIAS}

1. Moreno Domingo J, Lobato Micquelez JL, Arriba Olivenza $\mathrm{T}$, et al. Hiperplasia estromal pseudoangiomatosa de la mama (HEPA): estudio clínico, radiológico y patológico de 4 casos. Clin Invest Gin Obst 2012:39:253-9.

2. Milanezi MF, Saggioro FP, Zanati SG, Bazan R, et al. Pseudoangiomatous hyperplasia of mammary stroma associated with gynaecomastia. J Clin Pathol 1998;51:204-6.

3. Bolla D, Wiedemann N, Öhlschlegel C, Schöning A, Stickel J, Thürlimann $\mathrm{B}$, et al. Pseudoangiomatous stromal hyperplasia of breast in man: A case report. Breast J 2011;17:311-5.

4. Virk RK, Khan A. Pseudoangiomatous stromal hyperplasia. An overview. Arch Pathol Lab Med 2010;134:1070-4.

5. Celliers L, Wong DD, Bourke A. Pseudoangiomatous stromal hyperplasia: a study of the mammographic and sonographic features. Clin Radiol 2010;65:145-9.

6. Vuitch MF, Rosen PP, Erlandson RA. Pseudoangiomatous hyperplasia of mammary stroma. Hum Pathol 1986;17:185-91. 